\title{
Childhood Status Epilepticus: Current Status and Future Directions
}

\author{
VEena KaLRA \\ Senior Consultant, Indraprastha Apollo Hospitals, SaritaVihar, Delhi. \\ kalra.veena@gmail.com
}

S tatus epilepticus (SE) is a common neurological emergency of childhood with a high prevalence. Childhood status is unique in having a greater frequency of febrile status $(35 \%)$, recurrent status, and $30 \%$ risk of subsequent epilepsy [1]. SE has significant morbidity and mortality $(29 \%)$, which is influenced majorly by the etiology and the management. Given the lack of information on etiology and outcome of SE in children from India, the study by Chetan, et al. [2] in this issue of Indian Pediatrics is a welcome addition to the literature on the topic $[3,4]$.

Status epilepticus (SE) is a condition resulting from a failure of mechanisms that terminate a seizure or from strong seizure predisposition or irritative mechanisms which may be inflammatory, genetic, and cellular or subcellular, that perpetuate the seizure cycle. SE is often recurrent in epileptic children with structural malformations, resistant epilepsies, certain genotypes, recurrent inter-current illnesses and drug compliance issues.

The earlier definition of SE i.e., seizures persisting for 30 minutes with continuous motor activity or non-gain of consciousness between seizures has underwent significant changes.SE has now been stratified into Evolving SE, Established SE, Refractory SE, Super refractory SE, NORSE and FIRES, based on duration of seizures, response to one benzodiazepines and other antiseizure drugs [5]. This has resulted in clinical development of treatment guidelines and logical algorithms for domiciliary, community/hospital and tertiary level of care [6].

Benzodiazepines are the frontline agents for SE. They have a variety of routes of administration and quick onset of action - either of the rectal, oral, buccal, nasal, intramuscular and intravenous routes are possible. Rectal diazepam, buccal midazolam and oral lorazepam have now been superseded by intranasal midazolam. Intranasal midazolam exhibited best efficacy for non-venous treatment of SE in a recent meta-analysis [7,8].Early treatment of SE is the cornerstone of effective control and better outcomes [9]. Domiciliary therapy at seizure onset with intranasal midazolam is of supreme importance in halting the evolution of status and preventing established status, thus reducing cost of hospitalization and morbidity. This window of opportunity should not be missed. Empowerment of the family of a child with frequent seizures and of school health personnel with information regarding appropriate dose, route, technique and frequency of use of benzodiazepine is crucial. Its power is still not adequately harnessed in the community

At the hospital level,intravenous anti-seizure drugs are the mainstay [6]. The choice has really widened in the past decade from benzodiazepines, phenobarbitone, phenytoin and valproate to fosphenytoin, levetiracetam, lacosamide, steroids and immuno-suppressants. Multicentric studies are desired for comparative trials between the various conventional ASDs and the new anti-seizure drugs and to allocate their appropriate place. Two studies in this issue of Indian Pediatrics address this research need. Srivastava, et al. [10] report on the efficacy, serum levels achieved and side-effects after intravenous forphenytoin loading dose, and Vignesh, et al. [11] report on a randomized-control trial comparing phenytoin, valproate and levetiracetam in pediatric convulsive status epilepticus. At hospital level, every triage area and emergency should display the desired algorithm of use with doses, route of ASDs and the logical step-wise upgradation of therapy [12].

For refractory status and beyond, a clear guideline for ICU care in a tertiary setting is recommended.Patients must be referred on time.Patients with refractory status have multiple challenges and they merit monitoring of EEG, cardio-respiratory status, blood gas and recognition of cerebral edema. Familiarization with use of pentobarbitone, propofol, ketamine, and anesthetic agents is essential for their care in an ICU setting. The exhaustive review by Arya, et al. [13] in this issue of the journal shall definitely add to the pediatrician's and the intensivist's knowledge on the management of this 
vexing problem. Intravenous immunoglobulins for specific situations and ketogenic diet have found a unique place in SE management.

Novel researches in SE are now addressing the mechanisms of seizure initiation/persistence and will pave way for possible interventions [14,15]. This challenging research revealed the role of hypothermia in preventing neuronal death and hippocampal injury. Evidence of brain dynamics indicated reset after successful anti-seizure treatment of SE utilising stereo electrographic data (SEEG). In autoimmune SE, morphological alterations in microglia resulted in epileptogenesis. Studies in a kainite-induced SE model showed that neuronal loss does not necessarily correlate with higher seizure rate. Phytoalexins have shown efficacy in easing neurodegeneration, neuroinflammation, and aberrant neurogenesis, minimalizing ensuing chronic epileptic state. Ketogenic diet initiation in SE can help smoothen the withdrawal of aggressive therapy within 7-10 days, and is a useful adjunct in refractory SE.

Future research to reduce refractoriness of status and neuro-morbidity is warranted. The need to update management guidelines for pediatric SE for use in India, after incorporating recent evidence, is also highlighted. I look forward to translation of cutting edge research into clinical practice for the betterment of children with SE.

\section{REFERENCES}

1. Smith DM, McGinnes EL, Walleih DJ, Abend NS. Management of Status Epilepticus in children. J Clin Med; 2016;5:47.

2. Chetan C, Sharma S, Mathur SB, Jain P, Aneja S. Clinical profile and short-term outcome of pediatric status epilepticus at a tertiary-care center in Northern India. Indian Pediatr. 2020;57:213-7.

3. Gulati S, Kalra V, Sridhar MR. Status epilepticus in Indian children in a tertiary care centre. Indian J Pediatr. 2005; $72: 105-8$.
4. Sadik KC, Mishra D, Juneja M, Jhamb U. Clinicoetiological profile of pediatric refractory status epilepticus at a public hospital in India. J Epilepsy Res. 2019;9:36-41.

5. Trinka E, Cock H, Hesdorffer D, Rossetti AO, Scheffer IE, Shinnar S, et al. A Definition and Classification of Status Epilepticus - Report of the ILAE Task Force on Classification of Status Epilepticus. Epilepsia.2015; 56:1515-23.

6. Glauser T, Shinnar S, Gloss D, Alldredge B, Arya R, Bainbridge J, et al. Evidence-Based Guideline: Treatment of Convulsive Status Epilepticus in Children and Adults: Report of the Guideline Committee of the American Epilepsy Society. Epilepsy Curr. 2016;16:48-61.

7. Arya R, Gulati S, Kabra M, Sahu JK, Kalra V. Intranasal versus intravenous lorazepam for control of acute seizures in children - A randomised open label study. Epilepsia. 2011;52:788-93.

8. Arya R, Kothari H, Zhang Z, Han B, Horn PS, Glauser TA. Efficacy of nonvenous medications for acute convulsive seizures: A network meta-analysis. Neurology. 2015;85: 1859-68.

9. Eriksson K, Metsaranta P, Huhtala H, Auvinen A, Kuusela AL, Koivikko M. Treatment delay and the risk of prolonged status epilepticus. Neurology. 2005;65:1316-8.

10. Srivastava K, Bhartiya S, Gavli V, Patil R, Rajadhyaksha S. Efficacy, tolerability and serum phenytoin levels after intravenous fosphenytoin loading dose in children with status epilepticus. Indian Pediatr. 2020;57:218-21.

11. Vignesh V, Rameshkumar R, Mahadevan S. Comparison of phenytoin, valproate and levetiracetam in pediatric convulsive status epilepticus: A randomized double blind controlled clinical trial. Indian Pediatr. 2020;57:222-7.

12. Mishra D, Sharma S, Sankhyan N, Konkani R, Kamate M, Kanhere S, et al. Consensus Guidelines on Management of Childhood Convulsive Status Epilepticus. Indian Pediatr. 2014;51:975-90.

13. Samanta D, Garrity L, Arya R. Refractory and superrefractory status epilepticus. Indian Pediatr. 2020;57: 239-53.

14. Kirmani BF, Shetty AK, Shapiro LA. Editorial: New directions in the management of status epilepticus. Front Neurol. 2018;9:994.

15. Payne E. Status epilepticus - The hunt for treatable causes. Semin Pediatr Neurol. 2018;26:108-9. 